\title{
Sequence Variability of a Human Pseudogene
}

\author{
Rosa Martínez-Arias, Francesc Calafell, Eva Mateu, David Comas, Aida Andrés, \\ and Jaume Bertranpetit ${ }^{1}$
}

Unitat de Biologia Evolutiva, Universitat Pompeu Fabra, 08003 Barcelona, Spain

\begin{abstract}
We have obtained haplotypes from the autosomal glucocerebrosidase pseudogene ( $p s G B A$ ) for 100 human chromosomes from worldwide populations, as well as for four chimpanzee and four gorilla chromosomes. In humans, in a 5420-nucleotide stretch analyzed, variation comprises 17 substitutions, a 3-bp deletion, and a length polymorphism at a polyadenine tract. The substitution rate on the pseudogene $\left(1.23 \pm 0.22 \times 10^{-9}\right.$ per nucleotide and year) is within the range of previous estimates considering phylogenetic estimations. Recombination within the pseudogene was recognized, although the low variability of this locus prevented an accurate measure of recombination rates. At least $13 \%$ of the $p s G B A$ sequence could be attributed to gene conversion from the contiguous $G B A$ gene, whereas the reciprocal event has been shown to lead to Gaucher disease. Human psGBA sequences showed a recent coalescence time $(\sim 200,000 \mathrm{yr}$ ago $)$, and the most ancestral haplotype was found only in Africans; both observations are compatible with the replacement hypothesis of human origins. In a deeper timeframe, phylogenetic analysis showed that the duplication event that created psGBA could be dated at $\sim 27$ million years ago, in agreement with previous estimates.
\end{abstract}

In the last few years, studies on human genetic variation have undertaken the complete ascertainment of nuclear genomic sequences. The difficulty in ascertaining haplotypes in a diploid region has led the field first toward the study of X chromosome-linked regions, because haplotypes can be obtained directly from the amplification of X chromosomes in males (Zietkiewicz et al. 1997, 1998; Nachman et al. 1998; Harris and Hey 1999; Kaessman et al. 1999). Several studies to date have analyzed autosomal sequences in worldwide samples (Harding et al. 1997; Clark et al. 1998; Rana et al. 1999; Fullerton et al. 2000). Nevertheless, to the best of our knowledge, pseudogene sequences in humans have not yet been analyzed in worldwide samples.

The term "pseudogene" comprises a wide group of nonfunctional loci with a marked diversity of characteristics. They have been described as dead genes, because they are homologous to their functional source gene but contain nucleotide changes that prevent the production of a functional genetic product. Most pseudogenes are created by one of two mechanisms: tandem duplication or retrotransposition from a functional gene; however, more complex cases have been described (for review, see Cooper 1999). Tandem duplication originates nonprocessed pseudogenes, which are usually linked to their source gene and retain the exon-intron structure of the functional gene. Many duplications include the promoter region, and this allows some pseudogenes to be transcribed.

\footnotetext{
${ }^{1}$ Corresponding author.

E-MAIL jaume.bertranpetit@cexs.upf.es; FAX 34-93-542 2802. Article published on-line before print: Genome Res., 10.1101/gr.167701. Article and publication are at www.genome.org/cgi/doi/10.1101/ gr.167701.
}

One such tandem duplication originated the $G B A$ pseudogene ( $p s G B A)$, the nonfunctional duplicate of the $G B A$ gene, which encodes for the glucocerebrosidase protein (Expasy, EC 3.2.1.45). Mutations on GBA produce Gaucher disease (GD) (OMIM 230800, 230900, and 231000 for Gaucher type 1, type 2, and type 3 , respectively). More than 80,000 affected people in the world make GD the most prevalent lipid accumulation disorder.

GBA was mapped to 1q21 (Shafit-Zagardo et al. 1981; Devine et al. 1982; Ginns et al. 1985), and GBA cDNA was first cloned and sequenced from a fibroblast library (Sorge et al. 1985). The complete genomic sequences of $G B A$ and $p s G B A$ were described some years later (GenBank J03059 and J03060, respectively; Horowitz et al. 1989). The GBA gene is $7.6 \mathrm{~kb}$ long, and it is divided into 11 exons and 10 introns. psGBA is located $16 \mathrm{~kb}$ downstream from GBA (Zimran et al. 1990; Winfield et al. 1997); it contains the same exon and intron number and structure as $G B A$, although its length is $\sim 5.7 \mathrm{~kb}$ (Fig. 1). GBA is longer than psGBA because of several Alu insertions in intronic tracts of $G B A$ and a 55-bp deletion in exon 9 of psGBA (exon and intron notations in this report will follow the gene nomenclature). Despite the length difference, $p s G B A$ has maintained $96 \%$ sequence identity with the functional GBA gene. The high degree of sequence identity and the physical proximity between psGBA and $G B A$ allows gene conversion events from psGBA to $G B A$ (Hong et al. 1990; Latham et al. 1991), resulting in aberrant gene sequences that cause GD.

A rare trait of $p s G B A$ is that it is transcribed, because two TATA boxes and two CAT boxes in the $G B A$ promoter area are preserved in $p s G B A$, except for a sub- 

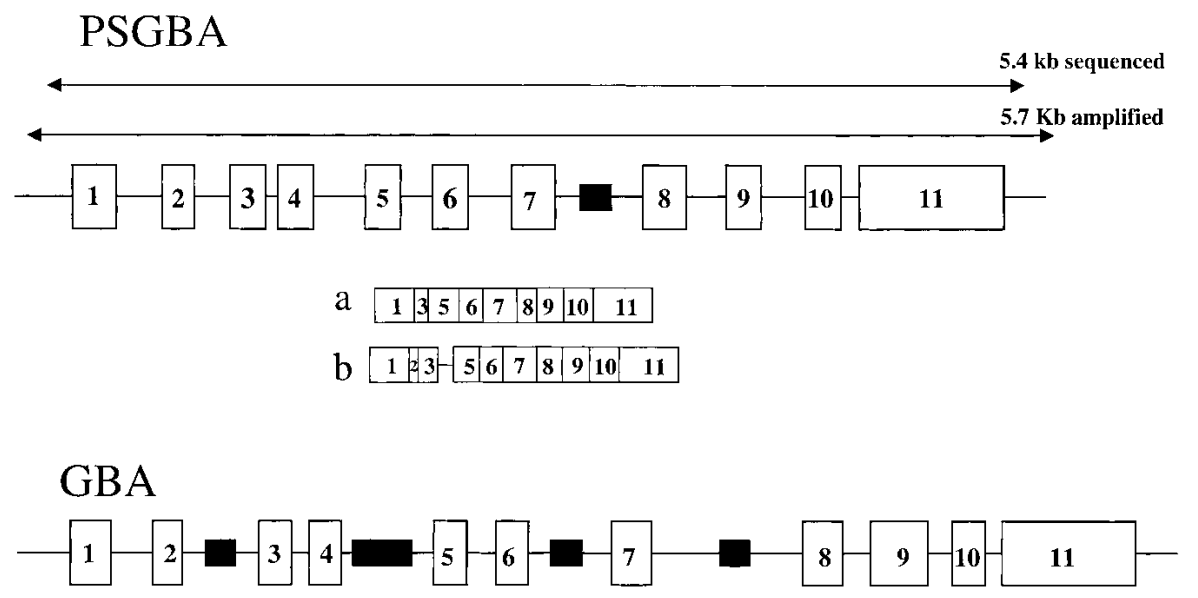

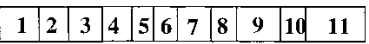

Figure 1 Schematic representation of $G B A$ and $p s G B A$. Exons are depicted as boxes with identification numbers inside. Alu sequences are represented as black boxes. The PCR-amplified and sequenced areas are indicated. The transcripts for both regions are represented with smaller boxes under each region: (a) psGBA transcript described by Sorge et al. (1990); (b) psGBA transcript described by Imai et al. (1993). The length of all tracts is roughly proportional to their actual nucleotide length.

stitution in the second CAT box (Horowitz et al. 1989). However, the activity of the $p s G B A$ promoter does not reach the levels of the $G B A$ active promoter (Reiner and Horowitz 1988). Two psGBA transcripts have been described (Fig. 1; Sorge et al. 1990; Imai et al. 1993).

The duplication within 1q21 is present in rhesus monkeys, and thus it may have occurred before the divergence of the great apes and Old World monkeys, 25 million yr ago (Mya). The presence in the two duplication copies of an Alu sequence of the Sx family, which is $40 \mathrm{My}$ old, places the age of duplication between 25 and 40 Mya (Winfield et al. 1997).

We analyzed sequence variation in $p s G B A$ in a sample of 100 worldwide chromosomes as a means to study variation in a human autosomal noncoding region. We have inferred the effects of recurrent mutation, recombination, and gene conversion on the phylogeny and polymorphism spectrum of this pseudogene. This is the first time that a wide spectrum of variability is reported for a completely noncoding autosomal tract, and that population genetic analyses are derived from a pseudogene.

\section{RESULTS}

\section{Nucleotide and Haplotype Diversity}

We ascertained 5420-bp psGBA haplotypes in $100 \mathrm{hu}$ man, four chimpanzee, and four gorilla chromosomes (GenBank AF267177, AF272642, and AF272641, for the human, chimpanzee, and gorilla sequences, respectively). One variable position and two haplotypes were detected in the two chimpanzee samples analyzed. As for the two gorilla samples, seven variable positions and three haplotypes were detected.

Among 100 human chromosomes analyzed, 18 variable sites, all of them diallelic, were identified, which corresponds to an average density of one segregating site in every 301 bp. Eleven individuals were homozygous for the whole tract analyzed. Only one deletion, of $3 \mathrm{bp}$, was detected. The other 17 variants were singlenucleotide substitutions. Among those, transitions were more frequent than transversions: 15 transitions $(88.2 \%$ of the single nucleotide substitutions) against two transversions $(11.8 \%)$. In addition to the 18 segregating sites, a short polymorphic polyadenine tract was found in position 3109, corresponding to the $A l u$ insertion in intron 7 . The exact number of adenines could not be read with certainty in the diploid sequences, but it was clear in the haploid sequences, in which alleles containing 9-11 repeats were detected. This site has not been included in the haplotype determination and further analysis because it follows different evolutionary patterns (i.e., mutation mechanisms and rates) than the rest of the variable sites. Seven of the 18 total segregating sites $(38.9 \%)$ are singletons (the rarer nucleotide variant appears once in the sample), and five (27.8\%) are doubletons (the rarer nucleotide variant appears twice in the sample).

None of the GBA gene counterparts of the polymorphic $p s G B A$ sites has been shown to be variable, except for nucleotide 4291: We have found a frequency of $6 \% \mathrm{~A}$ and $94 \% \mathrm{G}$ in $p s G B A$, and in the $G B A$ gene a frequency of $70 \% \mathrm{~A}$ and $30 \% \mathrm{G}$ was reported (Beutler et al. 1992). And, vice versa, the remaining polymorphisms reported in $G B A$ do not vary in $p s G B A$ in the present sample set. Site 4614 , a C/G polymorphism in humans, is also variable in gorillas, where A/G alleles were observed.

In humans, the 18 segregating positions define 25 different haplotypes (Table 1). Haplotype diversity was 0.853 . For the chimpanzee and gorilla alleles, only those positions that are polymorphic in the human sequence are shown in Table 1. Two major haplotypic groups are distinguishable in that table. Each group has a haplotype with high frequency (i.e., 3 and 17). Together, haplotypes 3 and 17 account for $52 \%$ of the total chromosomes. 


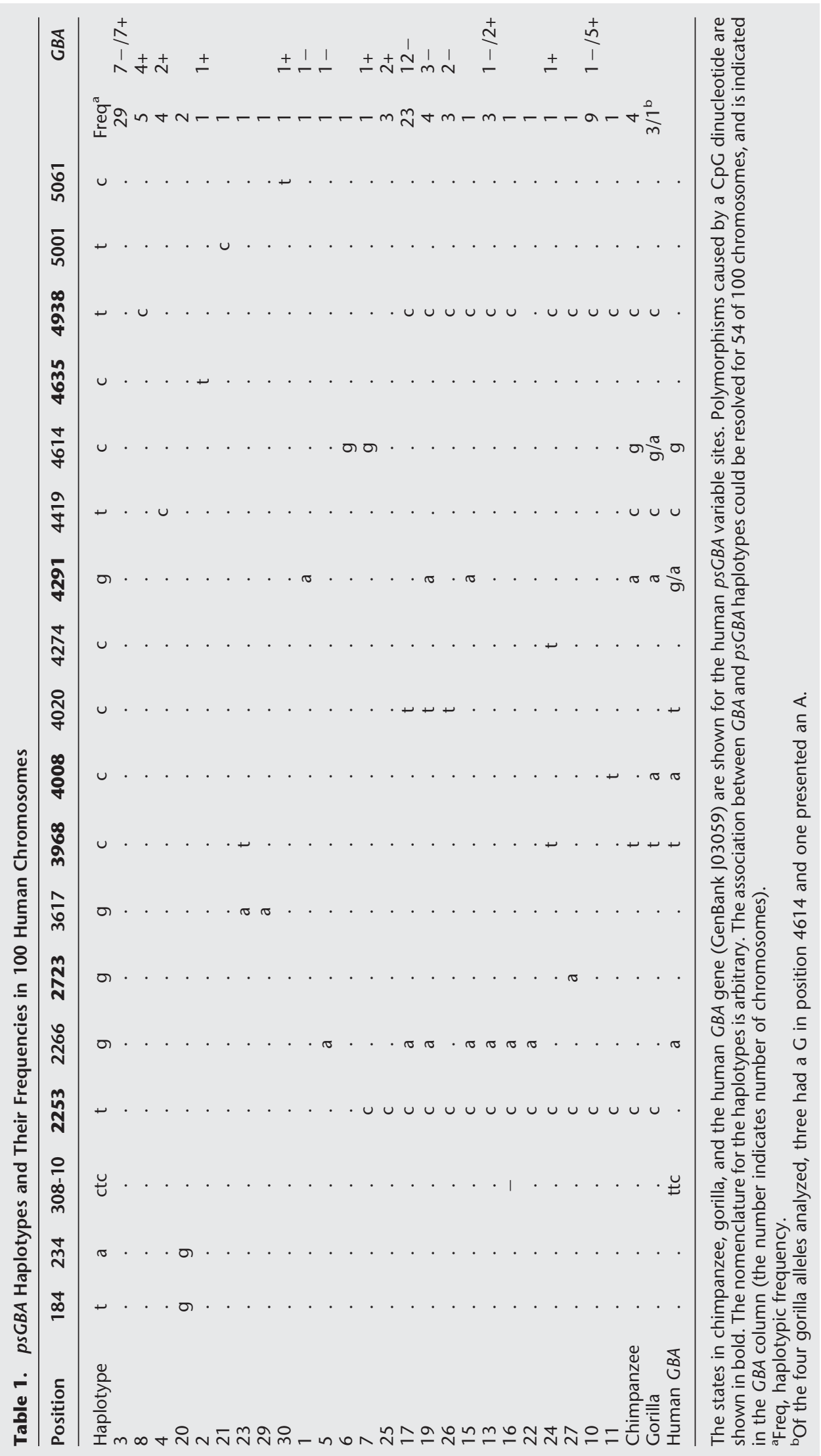


No clear geographic structure is observed in the distribution of human haplotypes (Table 2). To evaluate the effect of the differences among populations on the general variation, we calculated $F_{s t}$, which was $0.128(P<0.0001)$. This value is within the range for previous estimated $F_{s t}$ for mitochondrial DNA, Ychromosome, and autosomal polymorphisms, which indicates that most of the human genetic variation is due to differences within, rather than among, populations (Barbujani et al. 1997; Jorde et al. 2000).

Nucleotide diversity as the average heterozygosity, $\pi$, was 0.00044 (Nei and Li 1979). From $\pi$, the $\hat{\theta}_{\pi}$ estimator was 2.40 , which corresponds to the mean number of pairwise differences. The variability observed for psGBA is low in comparison to the values observed for other nuclear loci (Table 3 ). The $\hat{\theta}_{W}$ statistic is a different estimator of the variability that can be computed from the number of segregating sites (S) in a given locus (Watterson 1975), and was estimated as 3.28 for psGBA.

By the definition of a pseudogene, selection cannot act directly on it, but selective effects on neighboring genes can have a deep impact on sequence vari- ability in pseudogenes. Thus, the neutral model of evolution cannot be assumed for psGBA. Under the neutral model, both $\hat{\theta}_{\pi}$ and $\hat{\theta}_{W}$ estimate $4 \mathrm{~N}_{\mathrm{e}} \mu$, where $\mathrm{N}_{\mathrm{e}}$ is the effective population size and $\mu$ is the mutation rate. To test whether $p s G B A$ evolves according to the neutral model, we estimated Tajima's D statistic (Tajima 1989), which compares both $\hat{\theta}_{\pi}$ and $\hat{\theta}_{W}$ estimators, and it was -0.76 (not significant, $P>0.10$ ). The $\mathrm{D}^{*}$ and $\mathrm{F}^{*}$ statistics (Fu and Li 1993) can also be used to test whether mutations are selectively neutral. The $\mathrm{D}^{*}$ statistic is based on the differences between the number of singletons and the total number of segregating positions, and it was -1.33 (not significant, $P>0.10$ ). The $\mathrm{F}^{*}$ statistic is based on the differences between the number of singletons and $\hat{\theta}_{\pi}$, and it was found to be -1.34 for $p s G B A$ (not significant, $P>0.10$ ). The results of these tests do not allow us to reject a neutral model to explain the results.

\section{Polymorphism Patterns along psGBA: 5' and 3' Halves of the Pseudogene}

The number of segregating positions and the nucleotide diversity along $p s G B A$ are represented in Figure 2.

Table 2. Population Haplotype Frequencies

\begin{tabular}{|c|c|c|c|c|c|c|c|c|c|c|c|c|c|}
\hline $\mathrm{Hap}^{\mathrm{a}}$ & $\mathrm{BIA}^{\mathrm{b}}$ & TAN & SAH & BAS & CAT & DRU & YAK & $\mathrm{CHI}$ & MAY & NAS & SWA-EU & SSAFR & Total \\
\hline 3 & 1 & 4 & 2 & 2 & 4 & 3 & 6 & 3 & 4 & & 9 & 5 & 29 \\
\hline 17 & & 2 & 4 & 4 & 2 & 3 & 2 & 3 & & 3 & 9 & 2 & 23 \\
\hline 10 & 4 & 1 & 1 & 1 & & & & & & 2 & 1 & 5 & 9 \\
\hline 8 & 3 & & & & 2 & & & & & & 2 & 3 & 5 \\
\hline 4 & & & & & & & & & 4 & & & & 4 \\
\hline 19 & & & & & & 3 & 1 & & & & 3 & & 4 \\
\hline 13 & & & 2 & & & & & & 1 & & & & 3 \\
\hline 25 & & & & & & & & 2 & & 1 & & & 3 \\
\hline 26 & & & & & & & & & & 3 & & & 3 \\
\hline 20 & & & & 1 & & 1 & & & & & 2 & & 2 \\
\hline 5 & & & & & & & & & 1 & & & & 1 \\
\hline 1 & & & & & 1 & & & & & & 1 & & 1 \\
\hline 6 & & 1 & & & & & & & & & & 1 & 1 \\
\hline 7 & & 1 & & & & & & & & & & 1 & 1 \\
\hline 2 & & & & & & & & 1 & & & & & 1 \\
\hline 11 & & 1 & & & & & & & & & & 1 & 1 \\
\hline 15 & & & 1 & & & & & & & & & & 1 \\
\hline 16 & & & & 1 & & & & & & & 1 & & 1 \\
\hline 21 & & & & 1 & & & & & & & 1 & & 1 \\
\hline 22 & & & & & 1 & & & & & & 1 & & 1 \\
\hline 23 & & & & & & & 1 & & & & & & 1 \\
\hline 24 & 1 & & & & & & & & & & & 1 & 1 \\
\hline 27 & & & & & & & & & & 1 & & & 1 \\
\hline 29 & & & & & & & & 1 & & & & & 1 \\
\hline 30 & 1 & & & & & & & & & & & 1 & 1 \\
\hline $2 \mathrm{~N}^{\mathrm{c}}$ & 10 & 10 & 10 & 10 & 10 & 10 & 10 & 10 & 10 & 10 & 30 & 20 & 100 \\
\hline $\mathrm{k}^{\mathrm{d}}$ & 5 & 6 & 5 & 6 & 5 & 4 & 4 & 5 & 4 & 5 & 10 & 9 & 25 \\
\hline Private Hap ${ }^{e}$ & 2 & 3 & 1 & 2 & 2 & 0 & 1 & 2 & 2 & 2 & 5 & 4 & - \\
\hline
\end{tabular}

a Hap, haplotype.

'BIA, Biaka; TAN, Tanzanians; SAH, Saharawi; BAS, Basques; CAT, Catalans; DRU, Druze; YAK, Yakut; CHI, Chinese; MAY, Maya; NAS, Nasioi; SWA-EU, South West Asia and Europe (Basques, Catalans, and Druze); SSAFR, sub-Saharan Africans (Tanzanians and Biaka). ${ }^{\mathrm{c}} 2 \mathrm{~N}$, sample size in number of chromosomes.

${ }^{d} k$, number of different haplotypes found in each population.

ePrivate Hap, number of haplotypes found solely in that population. 
Table 3. Summary of Human Nuclear Sequence Variation Studies

\begin{tabular}{|c|c|c|c|c|c|c|c|}
\hline Authors & Year & Region & ${ }^{\mathrm{a}} \mathrm{L}$ & ${ }^{b} n$ & ${ }^{\mathrm{c}} \mathrm{S}$ & ${ }^{d} k$ & $\pi$ \\
\hline Hey & 1997 & PDHA1, X chr ${ }^{\mathrm{e}}$ & 1.8 & 8 & 4 & 4 & 0.0015 \\
\hline Zietkiewicz et al. & 1998 & Dystrophin, X chr & 7.6 & 250 & 35 & 36 & 0.0013 \\
\hline Nachman et al. & 1998 & Introns from 7 loci, $X \mathrm{chr}$ & 11.4 & 10 & 20 & - & 0.0008 \\
\hline Harris and Hey & 1999 & PDHA1, X chr & 4.2 & 35 & 25 & 11 & 0.0022 \\
\hline Kaessman et al. & 1999 & Non coding region, $\mathrm{X}$ chr & 10.2 & 69 & 33 & 20 & 0.0005 \\
\hline Jaruzelska et al. & 1999a & ZFX, X chr & 1.1 & 336 & 10 & 11 & 0.0011 \\
\hline Dorit et al. & 1995 & ZFY, Y chr & 0.7 & 38 & 0 & 1 & 0 \\
\hline Hammer & 1995 & YAP region, Y chr & 2.6 & 16 & 5 & 5 & 0.0004 \\
\hline Whitfield et al. & 1995 & SRY, Y chr & 18.3 & 5 & 3 & 4 & 0.0003 \\
\hline Jaruzelska et al. & 1999b & ZFY, Y chr & 0.7 & 205 & 1 & 2 & 0.00006 \\
\hline Shen et al. & 2000 & SMCY, UTY1, DBY, DFFY, Y chr & 81 & 53 & 98 & - & 0.000052 \\
\hline Li and Sadler & 1991 & 49 autosomal loci & 75 & 2 & - & - & $0.0011 \dagger$ \\
\hline Fullerton et al. & 1994 & $\beta$-globin, chr 11 & 3.1 & 36 & 17 & 17 & 0.0014 \\
\hline Harding et al. & 1997 & $\beta$-globin, chr 11 & 2.7 & 349 & 35 & 30 & 0.0018 \\
\hline Clark et al. & 1998 & LPL, chr 8 & 9.7 & 142 & 88 & 88 & 0.0020 \\
\hline Grimsley et al. & 1998 & HLA-H pseudogene, chr 6 & 0.3 & 34 & 15 & 11 & 0.0196 \\
\hline Rieder et al. & 1999 & $\mathrm{ACE}, \mathrm{chr} 17$ & 24 & 22 & 78 & 13 & 0.0009 \\
\hline Halushka et al. & 1999 & 75 genes & 190 & 149 & - & - & 0.0008 \\
\hline Rana et al. & 1999 & MC1R, chr 16 & 0.95 & 242 & 6 & 6 & 0.0020 \\
\hline Fullerton et al. & 2000 & ApoE, chr 19 & 5.5 & 192 & 22 & 31 & 0.0005 \\
\hline This study & 2001 & psGBA, chr 1 & 5.4 & 100 & 18 & 25 & 0.0004 \\
\hline
\end{tabular}

In the cases in which the $\mathrm{X}$ or the $\mathrm{Y}$ chromosomes were studied, the standardized $\pi$ (nucleotide diversity) is shown ( $\pi$ values were multiplied by $4 / 3$ or by 4 , respectively, to make them comparable to autosomes).

${ }^{a} \mathrm{~L}$, length per locus is indicated as number of kilobases sequenced.

${ }^{b} n$, number of chromosomes.

'S, number of segregating sites.

${ }^{d} k$, number of haplotypes observed.

${ }^{\mathrm{e}} \mathrm{chr}$, chromosome.

${ }^{\mathrm{f}}$ Nucleotide diversity for fourfold degenerate sites.

It can be seen that the segregating positions seem to concentrate toward the $3^{\prime}$ end of $p s G B A$, and nucleotide diversity does not appear to be homogeneous along the pseudogene.
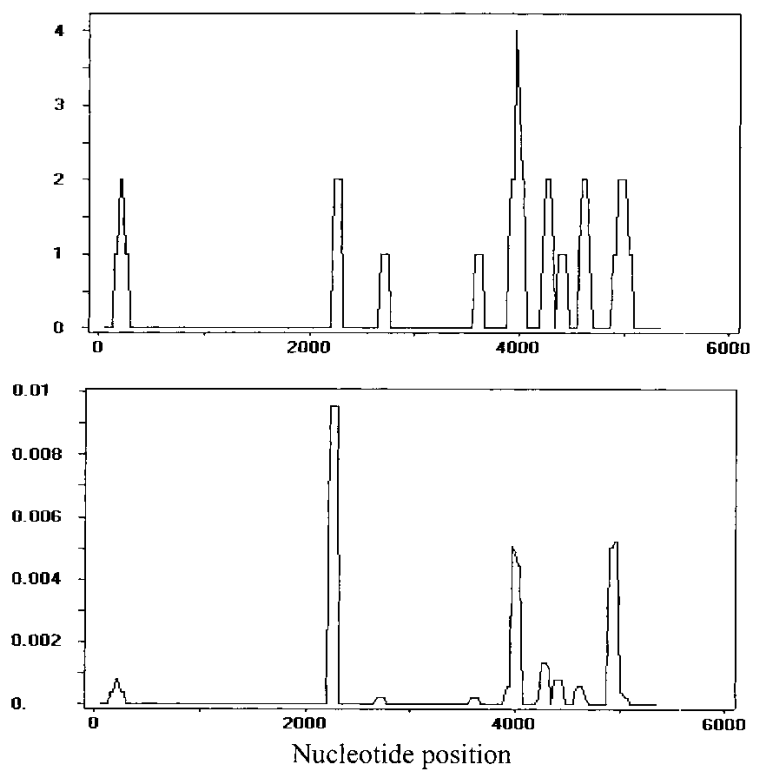

Figure 2 Number of segregating positions $(S)$, and nucleotide diversity $(\pi)$ along $p s G B A$. A window length of 100 nucleotides moved in steps of 25 nucleotides along the sequence was used.
In addition to the apparent nonuniform distribution of polymorphisms, a segment $\sim 2-\mathrm{kb}$ long in the $5^{\prime}$ half (1946 nucleotides, $35.9 \%$ of the total of nucleotides), from the CTC deletion in nucleotide 308 (exon 1) to the C/T polymorphism in nucleotide 2253 (exon 6), was found to lack any segregating site. Assuming that the distribution of polymorphic sites along the sequence is random, then the number of polymorphisms in a given segment would follow a Poisson distribution. The Poisson $\lambda$ parameter according to the observed proportion of polymorphic sites in the remaining sequence ( $3474 \mathrm{bp}$ ) is $\lambda=1.83$, and the probability of not observing any variable site in this $2-\mathrm{kb}$ stretch is $P=\mathrm{e}^{-\lambda}=0.16$. Therefore, the probability of absence of polymorphism in this region is not statistically significant and we cannot state that it evolves differently from the rest of the sequence. We can conclude, though, that if we had analyzed a shorter psGBA fragment, the results could have been biased.

The fact that 12 of the 17 segregating sites are placed in the $3^{\prime}$ half of the pseudogene made us check for possible differences in the variability between both halves of $p s G B A$. We considered as the $5^{\prime}$ half the first $2710 \mathrm{bp}$ and as the $3^{\prime}$ half the remaining $2710 \mathrm{bp}$. Six haplotypes and a nucleotide diversity of 0.00022 are observed in the $5^{\prime}$ half, against 16 haplotypes and a nucleotide diversity of 0.00066 in the $3^{\prime}$ half. The dif- 
ferent number of segregating positions in both halves of $p s G B A$ was tested with a $\chi^{2}$ test $\left(\chi^{2}=2.88,1\right.$ d.f., $P=0.090)$, and no significant differences were found.

A possible explanation for the levels of variation in a given genomic region may be that they are a function of the frequency of hypermutable $\mathrm{CpG}$ dinucleotides. To check whether that was the case for the two halves of psGBA, the possible differences between (1) the total number of CpG dinucleotides (48 in the $5^{\prime}$ half, 32 in the $3^{\prime}$ half) and (2) the number of mutated CpGs in both halves of the pseudogene (one in the $5^{\prime}$ half, six in the $3^{\prime}$ half) were tested with a $\chi^{2}$ test. No significant differences were found concerning the number of CpGs between the two psGBA halves $\left(\chi^{2}=3.20,1\right.$ d.f., $P=0.07$ ), but the number of mutated CpGs was significantly higher $\left(\chi^{2}=6.68,1\right.$ d.f., $\left.P=0.009\right)$ in the 3 ' half.

In summary, the apparent (but not statistically significant) difference in polymorphism between the 5' and $3^{\prime}$ moieties of $p s G B A$ seems to be due to the higher mutability of $\mathrm{CpG}$ dinucleotides in the $3^{\prime}$ half, which is the only significant difference between both halves.

\section{Substitution Rate}

We have estimated the substitution rate as the number of differences over $2 \mathrm{tL}$, $\mathrm{L}$ being the length of the segment compared and the divergence time between species. We assumed a 7-My divergence time between gorillas and humans, and gorillas and chimpanzees, and a 5-My divergence time between chimpanzees and humans. Average substitution rates of $1.30 \times 10^{-9}$, $1.43 \times 10^{-9}$, and $1.014 \times 10^{-9}$ per nucleotide and year were obtained for $p s G B A$ when comparing gorilla and human sequences (99 differences, 1.8\% divergence), chimpanzee and human sequences (78 differences, $1.4 \%$ divergence), and gorilla and chimpanzee sequences (77 differences, 1.4\% divergence), respectively. The mean weighted value for the substitution rate on $p s G B A$ is $1.23 \pm 0.22 \times 10^{-9}$.

The sequence of the $G B A$ gene in chimpanzee was obtained (GenBank AF285236), and this allowed us to estimate the substitution rate for the $G B A$ locus in the same way. As human $G B A$ we used the sequence on GenBank J03059. Five small indels and 62 substitutions (36 transitions and 26 transversions) were detected between chimpanzee and human sequences along 7156 nucleotides from the GBA gene. The substitution rate for $G B A$ between human and chimpanzee was estimated as $0.87 \pm 0.11 \times 10^{-9}$ per nucleotide and year. It should be noted that the confidence intervals for the substitution rates in the gene and in the pseudogene overlap slightly.

\section{Recombination and Recurrent Mutation}

psGBA is located in a centromeric area and, presumably, in a low-recombination genomic context. Nevertheless, some haplotypes present a pattern that could place the sequence in either of the two main groups observed among the haplotypes, that is, haplotypes 5 , 8 , and 25 and, to a lesser extent, 7, 22, and 1 (Table 1). This mixed pattern could be due to intragenic recombination, gene conversion, recurrent mutation, or back mutation events. In the absence of these processes, the maximum number of expected haplotypes for $\mathrm{S}$ diallelic segregating sites is $\mathrm{S}+1$ haplotypes. If $p s G B A$ has more alleles than would be expected from infiniteallele mutation alone, then at least one of these forces must have acted.

The absence of complete linkage disequilibrium can be also verified with the following rationale: If psGBA were in complete linkage disequilibrium, then we would expect that every haplotype constructed with the segregating sites only on the $3^{\prime}$ half of the locus would correspond to a single haplotype from the 5 ' half of the locus. On the contrary, six haplotypes are observed for the $5^{\prime}$ half and 16 for the $3^{\prime}$ half, whereas when considering the whole segment 25 haplotypes appear.

To measure the relevance of intralocus recombination events on $p s G B A$, the recombination parameter $\mathrm{C}=4 \mathrm{~N}_{\mathrm{e}} \mathrm{c}$, where $\mathrm{N}_{\mathrm{e}}$ is the effective population size and $c$ is the recombination rate per generation per base pair, was estimated. According to the estimation procedure suggested by Hudson (1987), in which the $\hat{C}$ estimator is based on the variance of the average number of nucleotide differences between pairs of sequences, $\hat{C}=16.9$. Nevertheless, this measure overestimates recombination if polymorphism is low and requires a long (more than 100-kb) segment, a large sample size, and high variability to be reliable (Hudson 1987). Even if the estimate was reliable, we should consider that possible recurrent mutational events are also counted as recombination events, and therefore recombination is overestimated. The $\gamma$ estimator of recombination (Hey and Wakeley 1997) does not depend on the polymorphisms in the sample and is less biased by sample sizes and shorter DNA length than Hudson's estimator. The $\gamma$ estimator was 1.271 for our sample set. From this estimate, the ratio of recombination events per mutation would be 0.39 (calculated as $4 \mathrm{~N}_{\mathrm{e}} \mu$ / $\left.4 \mathrm{~N}_{\mathrm{e}} \mathrm{c}=\hat{\theta}_{W} / \gamma\right)$. Nonetheless, the $\gamma$ estimator will yield an overestimate of recombination if there is homoplasy in the sample, as is probably the case for $p s G B A$.

The minimum number of recombination events along $p s G B A$ necessary to explain the observed variability (Hudson and Kaplan 1985) was estimated as four, between sites 2253 and 2266, 2266 and 4020, 4020 and 4291, and 4291 and 4938. However, the scarce number of segregating positions should make us be extremely cautious when trying to identify possible recombinant chromosomes. In fact, only four segregating positions separate haplotype 3 from haplotype 17 . In addition, two of the presumably most determinant 
positions in defining one or the other group (i.e., 2253, 2266, and 4938) are located on CpG dinucleotides (2253 and 4938), and therefore more than one mutational event could have originated the present observed variability at these positions. For example, it cannot be ascertained whether haplotype 8 was produced by a CpG recurrent mutation at 2253 or by a recombination event between haplotypes 3 and 10 .

To analyze further the origin of the possible recombinant alleles, we added data on three polymorphic sites analyzed for GBA (E. Mateu, F. Calafell, R. Martínez-Arias, A.Pérez-Lezaun, A. Andrés, J. Bertranpetit, unpubl.) to the haplotypic data for $p s G B A$. Twelve polymorphisms in tight linkage disequilibrium define two main haplotypes for $G B A$, named + and - , respectively, with frequencies $\sim 70 \%$ and $30 \%$ in Africans and Asians and the reciprocal in Europeans (Beutler et al. 1992; Glenn et al. 1994; E. Mateu, F. Calafell, R. Martínez-Arias, A. Pérez-Lezaun, A. Andrés, J. Bertranpetit, unpubl.). Haplotype ascertainment from genotype data showed a significant linkage disequilibrium between haplotypes - and 17, and + and 3 . Those haplotypes that were double haplotype homozygotes for $G B A$ or $p s G B A$ allowed unambiguous phase resolution and determination of the joint $G B A-p s G B A$ haplotypes. From those, out of 14 chromosomes with psGBA haplotype 3, 7 were linked to $G B A$ haplotype and 7 to $G B A$ haplotype + . The 12 resolvable $p s G B A$ haplotype 17 chromosomes were all linked to $G B A$ haplotype - .

In the same way, haplotypes 25 and 7 were linked to $G B A$ haplotype +. These two haplotypes have a $\mathrm{C}$ in position 2253 and therefore could be placed at first sight, erroneously, within haplogroup 17 (if position 2253 was taken as diagnostic). However, because $G B A$ haplotype + seems not to be linked with haplogroup 17 , it seems more likely that these are not recombinant haplotypes between haplotype group 17 and group 3 chromosomes, but rather that they have not yet lost $2253 \mathrm{C}$ through repeated mutation at that CpG dinucleotide.

On the whole, it seems that recombination is low, although not absent, at $p s G B A$ and that other forces such as gene conversion and recurrent or back mutation may have had a prominent role in shaping the variability spectrum of $p s G B A$.

\section{Interlocus Gene Conversion}

We aligned chimpanzee psGBA, human $p s G B A$, and human $G B A$ sequences (GenBank AF272642, AF267177, and J03059, respectively) to detect possible interlocus gene conversion events (gene conversion between different alleles at different loci) between psGBA and $G B A$ and to assess their magnitude. Next, we added the sequence of the $G B A$ gene in chimpanzee (GenBank AF285236) to detect the possible influence of gene conversion events in chimpanzees on those fragments where gene conversion in humans would be likely, and also to have an external reference for the $G B A$-specific sequence pattern. We would detect gene conversion as a string of nucleotide positions placed on a different haplotype background. We looked for gene-specific patterns in the pseudogenes, for pseudogene patterns in the genes, and also for nucleotide positions that interrupted those patterns (Fig. 3).

When we compare the $G B A$ and $p s G B A$ sequences in humans and chimpanzees, some tracts seem to be the clear result of gene conversion events from the human GBA gene to human psGBA. These are the fragments from 439 to 567,1264 to 1265,1628 to 1682 , 1884 to 1982,2105 to 2241,4204 to 4261 , and 4680 to 4910 (the numeration of human $p s G B A$ reported here is used [GenBank AF267177]). In these tracts, at least in two consecutive positions, human psGBA has the same pattern as human $G B A$, and it is different from psGBA in chimpanzee (to which it should be more similar). In addition, the gene pattern is the same in $G B A$ from chimpanzee, while the characteristic pseudogene pattern would have been preserved only in $p s G B A$ in chimpanzee.

In other tracts, human $G B A$ is the locus with a distinct sequence, and the sequence from chimpanzee $G B A$ seems to have acquired the pattern from chimpanzee psGBA, such as in fragment 1295-1324. However, we have analyzed $G B A$ in chimpanzee only for those positions that would allow us to recognize gene conversions in humans, and we cannot identify with certainty gene conversion tracts elsewhere in the chimpanzee sequences.

There is a third kind of tracts, in which $p s G B A$ and $G B A$ sequences are equal within each species but different across species. These fragments are all located in introns, so that if gene conversion had occurred in both species, the direction could not be unequivocally established. Besides, recurrent or parallel mutations could have taken place, which could be mistaken for gene conversion.

Taking into account only the first type of fragments, in which gene conversion is more obvious, at least $709 \mathrm{bp}$ of $p s G B A$ sequence (13\% of the total length) is affected by this phenomenon. The high sequence similarity among the four sequences makes it difficult to pinpoint the extent of gene conversion, and therefore this is a minimum estimate, because gene conversion could extend longer and go unrecognized because of the high sequence similarity between gene and pseudogene. Gene conversion was random with respect to exon-intron distribution $\left(\chi^{2}=1.216,1\right.$ d.f., $P=0.27)$.

Recent gene conversion events may not have reached fixation in the population and remain polymorphic at $p s G B A$. That could be the case for positions 


\begin{tabular}{|c|c|c|c|c|c|c|c|c|c|c|c|c|c|c|c|c|c|c|c|c|c|c|c|c|}
\hline$p s G B A n t$ & 184 & 234 & 259 & 300 & 308 & 439 & 567 & 592 & 614 & 627 & 849 & 974 & $1137^{*}$ & 1153 & 1264 & $1265 i$ & 1295 & 1324 & 1414 & 1415 & 1628 & 1659 & 1674 & 1682 \\
\hline GBA chimp & c & a & $T$ & g & tte & $\mathrm{T}$ & G & G & A & A & G & g & c & $t$ & c & 0 & C & C & C & a & G & G & c & $\mathrm{C}$ \\
\hline psGBA chimp & $t$ & a & G & c & ctc & C & A & G & $\mathrm{C}$ & A & G & $\mathrm{c}$ & c & a & G & c & C & C & $\mathrm{T}$ & g & A & A & $\mathrm{T}$ & A \\
\hline GBA human & c & a & $T$ & g & ttc & $T$ & G & $\mathrm{T}$ & A & G & C & g & 0 & $t$ & C & 0 & $\mathrm{~T}$ & G & C & a & G & G & c & C \\
\hline psGBA human & $g / t$ & $g / t$ & $\mathrm{~T}$ & c & ctc- 0 & $\mathrm{~T}$ & G & G & A & A & c & c & 0 & a & C & 0 & $\mathrm{C}$ & $\mathrm{C}$ & $\mathrm{C}$ & g & G & G & c & $\mathrm{C}$ \\
\hline
\end{tabular}

\begin{tabular}{|c|c|c|c|c|c|c|c|c|c|c|c|c|c|c|c|c|c|c|c|c|c|c|c|c|c|c|c|}
\hline$p s G B A n t$ & 1727 & 1798 & 1826 & 1851 & 1884 & 1982 & 1994 & 2043 & 2089 & 2105 & 2190 & 2216 & 2241 & 2253 & 2266 & 2275 & 2525 & 2554 & 2671 & 2723 & 3617 & 3678 & 3686 & 3851 & 3968 & 3970 & 3982 \\
\hline GBA chimp & c & G & C & $c$ & C & $T$ & $\mathrm{~T}$ & G & G & C & C & c & A & $t$ & a & $t$ & G & $t$ & c & g & g & C & $t$ & C & $t$ & C & A \\
\hline psGBA chimp & $t$ & A & c & $t$ & $\mathrm{~T}$ & C & $\mathrm{T}$ & G & G & G & $\mathrm{T}$ & $\mathrm{T}$ & $\mathrm{T}$ & C & G & g & A & c & G & g & \& & A & c & $\mathrm{T}$ & $T$ & $\mathrm{C}$ & $\mathrm{T}$ \\
\hline GBA human & $\mathrm{c}$ & G & $T$ & $\mathrm{c}$ & c & $\mathrm{T}$ & G & C & A & C & C & C & A & $\mathrm{T}$ & A & 1 & G & c & C & g & g & c & $t$ & C & $\mathrm{T}$ & $\mathrm{T}$ & A \\
\hline psGBA human & $t$ & G & T & $t$ & C & $T$ & G & C & G & c & C & C & A & $\mathrm{C} / \mathrm{T}$ & $\mathrm{A} / \mathrm{G}$ & $g$ & G & 1 & c & $\mathrm{a} / \mathrm{g}$ & $\mathrm{a} / \mathrm{g}$ & C & c & C & $\mathrm{C} / \mathrm{T}$ & $\mathrm{T}$ & A \\
\hline
\end{tabular}

psGBAnt $\quad 400840154020408241044204426142744291435244194584 \quad 4614 \quad 4635468047354868-744908-104928493850015061$

\begin{tabular}{|c|c|c|c|c|c|c|c|c|c|c|c|c|c|c|c|c|c|c|c|c|c|c|}
\hline GBA chimp & a & G & $t$ & c & g & c & c & c & a & C & c & G & g & c & $\mathrm{T}$ & $\mathrm{T}$ & cectgg & $\operatorname{ctgt}$ & G & c & $t$ & $\mathrm{c}$ \\
\hline psGBA chimp & $\mathrm{c}$ & $\mathrm{C}$ & c & $\mathrm{T}$ & c & $\mathrm{T}$ & G & c & $\mathbf{a}$ & $\mathrm{T}$ & $\mathrm{c}$ & A & $g$ & c & $\mathrm{C}$ & $\mathrm{C}$ & 0 & 0 & G & c & 1 & c \\
\hline GBA human & $\mathbf{a}$ & G & $t$ & c & $g$ & c & c & c & $\mathrm{a} / \mathrm{g}$ & C & c & G & $g$ & c & $T$ & $T$ & gectgg & ctgt & G & $t$ & $t$ & c \\
\hline psGBA human & $c / t$ & G & $c / t$ & c & c & c & c & $\mathrm{c} / \mathrm{t}$ & $\mathrm{a} / \mathrm{g}$ & c & $\mathrm{c} / \mathrm{t}$ & G & $\mathrm{c} / \mathrm{g}$ & $c / t$ & $\mathrm{~T}$ & $\mathrm{~T}$ & gectgg & etgl & A & $c / t$ & $c / t$ & $\mathrm{c} / \mathrm{t}$ \\
\hline
\end{tabular}

Figure 3 Gene conversion tracts along psGBA. Positions at which both psGBA from chimpanzee and human differ and human $G B A$ and ps $G B A$ are equal are considered possible gene conversion fragments from GBA to psGBA (light shading). The position following one of these blocks that was equal between both pseudogenes and different with the GBA gene (so that it interrupts the possible tract of gene conversion) is also indicated in the figure in lower case. Dark shadows indicate tracts where gene and pseudogene sequences are equal both in humans and in chimpanzees, but where orthologous sequences are different across species. Positions at which human ps $B A$, human $G B A$, and chimpanzee psGBA were different, or at which the GBA gene and psGBA in chimpanzee are equal and different from human psGBA, have not been taken into account. Variable sites found in the human pseudogene are represented in bold. (*) Similarity between genes and pseudogenes in position 1137 has to be taken with caution, because it is located after a single-nucleotide run of seven adenines, and the similarity could be a product of polymerase slippage and not of gene conversion. $1265 \mathrm{i}$ indicates the insertion after site 1265 in human $p s G B A$.

$2253,2266,4020$, and 4938 , because on them one allele corresponds to the state in psGBA in chimpanzee and the other variant to the state in the human $G B A$. Polymorphisms on sites 2253 and 4938 are located on a hypermutable $\mathrm{CpG}$, which could as well account for the changes from $G$ to $A$ in this position.

\section{Haplotype Phylogeny}

A network with all possible phylogenetic links among haplotypes was constructed with the Network v, 2.0b software (Fig. 4). Reticulations in this median network can reveal homoplasy and possible recombination (Bandelt et al. 1995). In particular, three of the four estimated recombination events (between sites 2253 and 2266, 2266 and 4020, and 4020 and 4291) are reflected as reticulations in the network.

Two major clades are observed, with centers in the two most frequent haplotypes, namely 3 (in 29 chromosomes) and 17 (in 23 chromosomes). The phylogenetic structure around haplotype 3 is clearly starlike, with 10 haplotypes radiating directly from it. No such starlike structure is observed around haplotype 17.

The extent of the phylogenetic separation between the haplotypes radiating from haplotypes 3 or 17 was ascertained by means of their pairwise difference distributions. Figure 5 shows the overall pairwise difference distribution, as well as the pairwise difference distributions within each haplotype group and between them. A slightly bimodal curve can be appreciated, which is caused by differences within (left-hand peak) and between (right-hand peak) haplotype groups 3 and 17 , as shown by the separate pairwise distributions.

\section{Time to the Most Recent Common Ancestor and Mutation Ages}

To infer mutational ages and thus put the haplotype phylogeny in a historical frame, the method based on coalescence theory suggested by Griffiths and Tavaré (1994, 1998a, 1998b) was applied. Coalescence theory provides a mathematical tool for inferring backward in time the genealogy of genes or alleles sampled from a present population.

The ancestral state for the human psGBA was inferred as the state at each polymorphic site that would give the most parsimonious $p s G B A$ phylogeny, rooted with the chimpanzee psGBA. Human ancestral states for almost all polymorphic sites match those in the chimpanzee sequence, except for four positions (3968, 


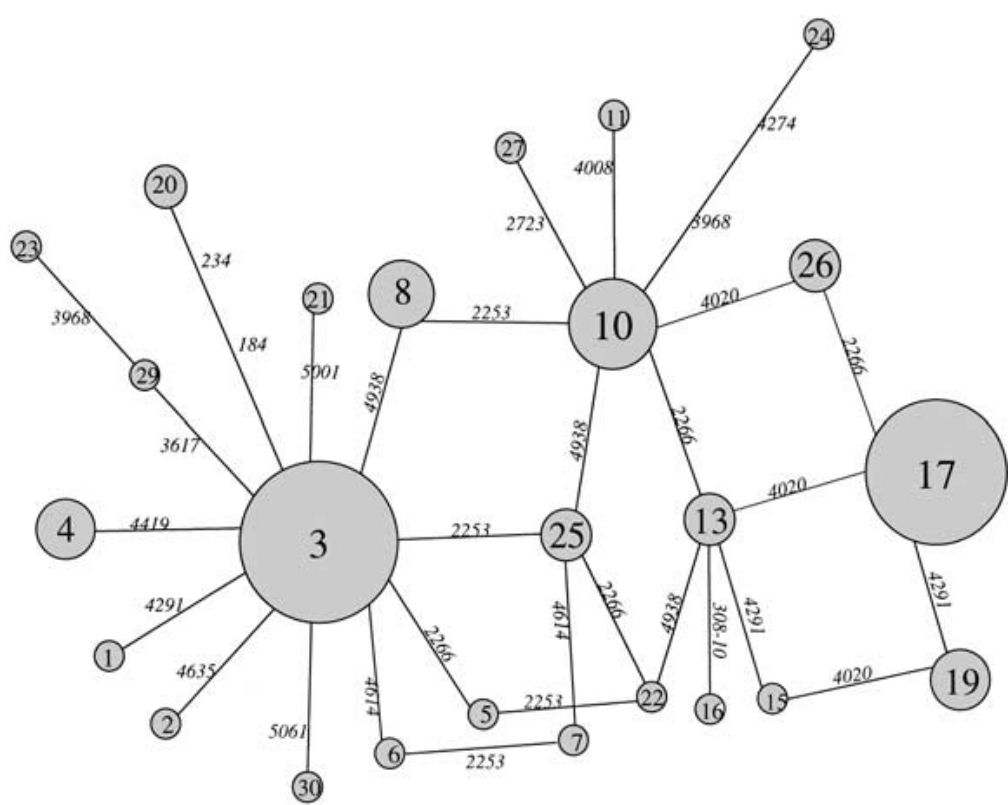

Figure 4 Median network constructed from the 25 haplotypes in our sample. Haplotype number is shown inside the circles, and mutation positions are indicated on the branches linking two haplotypes. Circle areas are proportional to the frequency of the haplotypes. Branch lengths are proportional to the number of mutational events they represent.

4291, 4419, and 4614), in which the nucleotide states present in chimpanzee are scattered on eight haplotypes located in external branches of the human network.

Mutation ages were estimated using the maximum-likelihood estimate of $\theta, \hat{\theta}_{\mathrm{ML}}$ (4.45), which is the $\theta$ value that would yield the most likely coalescent tree. $\hat{\theta}_{\mathrm{ML}}$ was calculated using the GENETREE program. Ef-

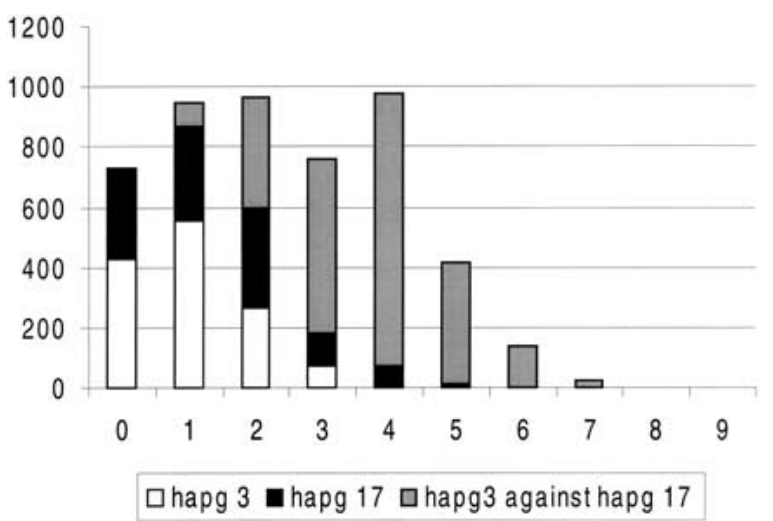

Figure 5 Pairwise nucleotide difference distribution. The differences within haplogroup (hapg) 3, within haplogroup 17, and the differences resulting from the comparison between both haplogroups are shown. Haplogroup 3 comprised haplotypes 1, 2, 3, $4,5,6,7,8,20,21,23,25,29$, and 30, and haplogroup 17 comprised haplotypes 10, 11, 13, 15, 16, 17, 19, 22, 24, 26, and 27. fective population size was estimated as $\mathrm{N}_{\mathrm{e}}=8345$ from $\theta=4 \mathrm{~N}_{\mathrm{e}} \mu$, using the $\hat{\theta}_{\mathrm{ML}}$ estimate and the previously calculated substitution rate per sequence and generation, $\mu=13.33 \times 10^{-5}$. Under these conditions, and assuming neutrality, the infinite-sites mutation model (haplotypes presumably affected by recurrent mutation or recombination were omitted from the analysis), random mating, no population substructure, constant population size, and a generation time of $20 \mathrm{yr}$, the coalescent time, or time to the most recent common ancestor (TMRCA) was estimated at $199,000 \pm 58,600$ yr (Fig. 6). Diversity data were estimated for the haplotypes compatible with the infinite-sites mutation model and were not significantly different from those estimated from the complete set of haplotypes, so that no major biases were introduced when computing the mutation ages. The age of the mutations in the gene genealogy range from 163,800 to $5170 \mathrm{yr}$ (Table 4).

Mutation 4938 would lead to haplotype 3 and the group of haplotypes radiating from it, and it has an estimated age of $\sim 74,400 \pm 26,600 \mathrm{yr}$. To assess the reliability of the estimated ages, an independent estimate of the age of haplogroup 3 was calculated according to a Poisson distribution of mutations, as explained in Methods section. Haplotype 3 was considered to be the ancestral sequence of haplogroup 3, which was designed as those haplotypes that could be derived unequivocally from haplotype 3 , that is, $2,4,6,20,21,29,30$, and haplotype 3 itself. Age of haplogroup 3 can be inferred as $43,000 \pm 11,900 \mathrm{yr}$, which overlaps with the previous estimate.

We also computed the TMRCA assuming a population growth model instead of a population with constant size. Growth parameter $\hat{\beta}_{\mathrm{ML}}$ and $\hat{\theta}_{\mathrm{ML}}$ under an exponential growth model were estimated simultaneously with the GENETREE program. We assumed the same growth rate for all populations. We obtained a value of $\hat{\beta}_{\mathrm{ML}}$ of 11.4 and of $\hat{\theta}_{\mathrm{ML}}$ of 8.6 . From this $\hat{\theta}_{\mathrm{ML}}$ value we obtain an effective population size of 16,125 individuals. TMRCA and mutation-age estimates obtained under a population growth model were markedly lower than those obtained under a model of constant population size (Table 4). The general pattern of the gene tree obtained under a growth model is the same as under a constant population model, except that branches have been shortened between mutations 2266 and 3968 and between 4938 and 3968. The difference in the likelihood of the trees under constant and growth models was not statistically significant. 


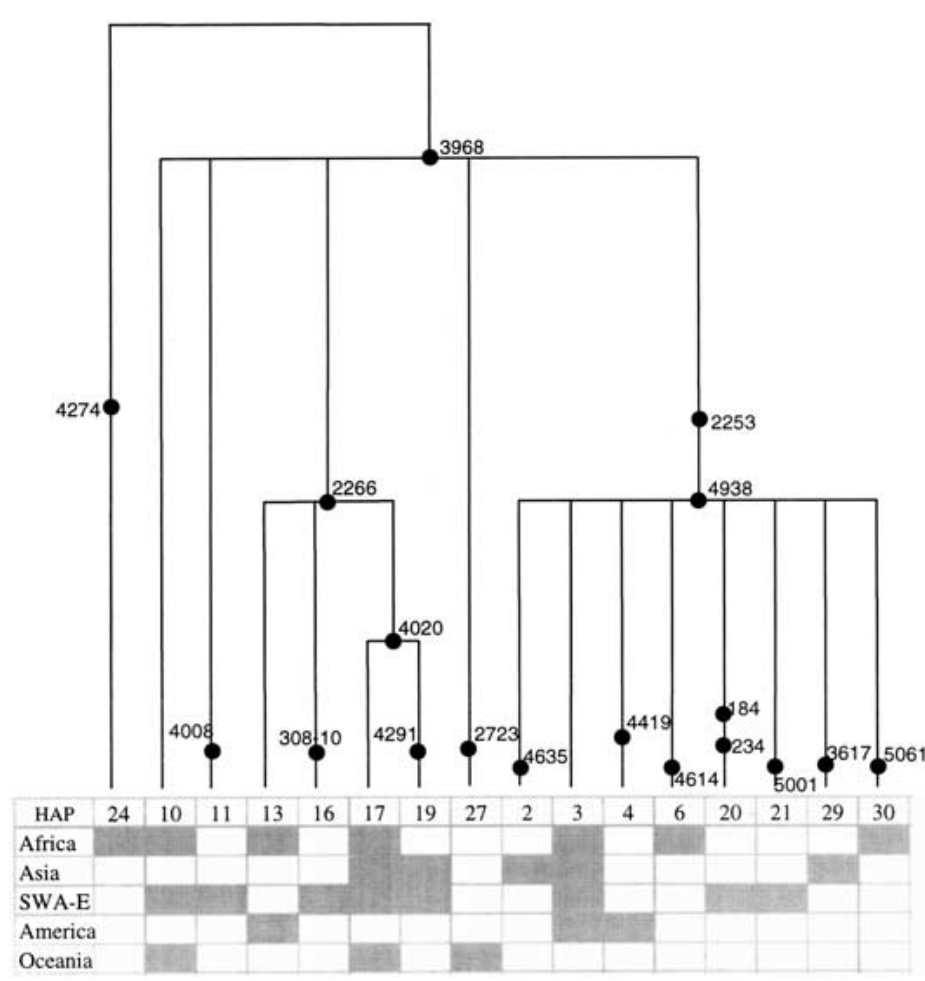

Figure 6 Coalescent tree of the psGBA haplotypes, for the psGBA haplotypes compatible with the infinite-sites mutation model. The tree was computed using $\hat{\theta}_{\mathrm{ML}}=4.45, \mathrm{~N}_{\mathrm{e}}=8345, \mu=13.33 \times 10^{-5}$ per generation and year, and a 20 -yr generation time, through $2 \times 10^{7}$ iterations. Numbers along branches represent mutational events. The continents where the haplotypes are present are indicated. (SWA-E) Southwest Asia and Europe.

\section{Phylogenetic Relations among Orthologous and Paralogous Sequences}

The neighbor-joining phylogenetic algorithm (Saitou and Nei 1987) was applied to distances between pairs of sequences estimated with the Kimura 2-parameter method. To assess the reliability of branching, 1000 bootstrap replicates were performed. Trees were constructed among the human $p s G B A$ haplotypes, among all the pseudogene sequences (human, chimpanzee, and gorilla), and among those and the GBA gene sequences in human (GenBank J03059) and chimpanzee (GenBank AF285236) (Fig. 7).

It is clear from Figure 7 that the duplication event that created $p S G B A$ preceded hominoid speciation, because the $G B A$ and $p s G B A$ sequences cluster clearly by homology rather than by species. The time of duplication for $p s G B A$ was calculated from the differences between $G B A$ and $p s G B A$ in humans as number of differences over $L \mu, L$ being the length compared between two sequences, and $\mu$ the sum of the estimated substitution rates for $G B A$ and $p s G B A$, because the divergence in the two branches after the duplication, for $G B A$ and for $p s G B A$, has to be considered. The estimate was 23.4 My with the human data and 23.2 My with the chimpanzee data.

\section{DISCUSSION}

We have sequenced an $\sim 5.5-\mathrm{kb}$ stretch containing pseudogene $p s G B A$ in 100 chromosomes distributed among all major world geographic areas and found that $p s G B A$ has the lowest nucleotide diversity observed for an autosomal locus. On average, two randomly chosen sequences of nearly $5.5 \mathrm{~kb}$ will be different at about two nucleotide sites. This low value was not expected for a noncoding region such as a pseudogene, which would be seemingly free to accumulate variation unchecked by purifying selection. Next, we discuss the main evolutionary forces that may have acted on $p s G B A$ to create and shape genetic variation, as well as the inferences that can be drawn from that variation both on the evolutionary history of the region and on human evolution.

\section{Genomic Forces Acting on psGBA: Mutation, Recombination, Gene Conversion}

The substitution rate we have found in $p s G B A$ is not higher than the substitution rates described for functional genes. Substitution rate values for psGBA and GBA are indeed close. This fact might be taken into account when considering pseudogenes to estimate the rate of spontaneous mutation. The present results indicate a large heterogeneity in pseudogene mutation rates, as lower values than those considered "neutral" have been found in a clearly nonfunctional genomic region. Previous estimates of substitution rates should be taken with caution because they were estimated from a limited number of pseudogenes that have not been proven not to be under selective constraints ( $\mathrm{Li}$ et al. 1981).

As shown by the different number of $\mathrm{CpG}$ dinucleotides that are found to be polymorphic in the $5^{\prime}$ and $3^{\prime}$ moieties of $p s G B A$, mutation does not seem to have a homogeneous action along the pseudogene. Moreover, a phylogenetic network of psGBA haplotypes showed instances of repeated mutations at some sites, although most of $p s G B A$ is fixed.

The nonsignificance of Tajima and $\mathrm{Fu}$ and $\mathrm{Li}$ tests does not allow us to directly reject neutrality for psGBA. However, it does not imply absence of selection, either. It might be that to detect the effect of selection on psGBA these tests would require a larger sample size (Simonsen et al. 1995). Besides, the variability observed for $p s G B A(\pi=0.00044)$ is low in comparison to the values observed for other autosomal loci, with nucleotide diversity values that range from 0.0196 for the HLA-H pseudogene (Grimsley et al. 1998) to 0.0005 for the Apolipoprotein E gene (Fullerton et al. 2000). The nucleotide diversity of $p s G B A$ is lower than for all autosomal coding regions. Although 
Table 4. Time to Most Recent Common Ancestor (TMRCA) and Ages of the Deepest Mutations in the psGBA Phylogeny

\begin{tabular}{lcccc}
\hline $\begin{array}{l}\text { Mutation } \\
\text { and TMRCA }\end{array}$ & $\begin{array}{c}\text { Age, } \\
\text { constant } \\
\text { population }\end{array}$ & $\begin{array}{c}\text { SD, } \\
\text { constant } \\
\text { population }\end{array}$ & $\begin{array}{c}\text { Age, } \\
\text { exponential } \\
\text { population growth }\end{array}$ & $\begin{array}{c}\text { SD, } \\
\text { exponential } \\
\text { population growth }\end{array}$ \\
\hline TMRCA & 199,000 & 58,600 & 91,000 & 17,000 \\
3968 & 163,800 & 48,400 & 81,600 & 13,900 \\
4274 & 99,460 & 67,500 & 44,800 & 24,400 \\
2253 & 95,000 & 32,700 & 58,000 & 11,400 \\
4938 & 74,400 & 26,600 & 49,900 & 10,100 \\
2266 & 74,000 & 28,300 & 53,200 & 12,000 \\
\hline
\end{tabular}

Times have been computed under constant population size and exponential growth models. Ages are given in years and have been calculated with a generation time of $20 \mathrm{yr}$, a population size of 7330 individuals under the constant population model, and a population size of 14,170 individuals under the exponential population growth model.

the standard tests for neutral evolution were not statistically significant, this low diversity could indicate selection having an effect on $p s G B A$.

Gene conversion seems to have played an important role in the evolution of $G B A$ and $p s G B A$, because sequences at several tracts are probably due to this mechanism. Gene conversion is a homogenizing mechanism between homologous loci in the genome. It consists of a nonreciprocal transfer of information: An allele (information acceptor) is modified by a second allele (information donor) that remains unchanged. The length of the DNA segment converted can vary from a few base pairs to several hundreds. Gene conversion cannot be proved without ambiguity because its result is not distinguishable from a double crossing-over event. However, the probability of a double crossing-over event in a tract shorter than several hundred kilobases is extremely low (Broman and Weber 2000). Thirteen percent of the $p s G B A$ sequence probably has its origins on $G B A$. These ancient gene conversion tracts are fixed on $p s G B A$; they may have happened well before the TMRCA of the current haplotypes, and therefore they do not have any effect on the observed variability. The fact that the gene conversion tracts detected are random with respect to exonintron distribution was expected, because the transference of any DNA fragment to $p s G B A$ does not have functional implications. We cannot discard recent gene conversions as the cause for some of the segregating positions, that is, 2253, 2266, 4020, and 4938. Recurrent mutation due to the hypermutability of CpG dinucleotides could as well account for the mutations 2253 and 4938.

It might be worth noting that we have detected gene conversion from $G B A$ (under selective pressure) to psGBA (nonfunctional, and therefore presumably without purifying selection), but not the other way around. Gene conversions from $p s G B A$ to $G B A$ happen indeed and have been detected, because the individuals carrying those converted alleles are affected with GD (Ko- privica et al. 2000; Stone et al. 2000). However, these individuals have a low fitness ( $G B A$ alleles with $p s G B A$ tracts interrupting the reading frame are lethal in homozygosity), and these $G B A$ alleles are either not passed on to the next generation or are lost slowly over time because of purifying selection. Thus, the detailed knowledge of sequence variation at $p s G B A$ may be crucial for recognizing $p s G B A$ to $G B A$ gene conversion events in GD chromosomes.

\section{The GBA Region Phylogeny}

When $p s G B A$ and $G B A$ sequences from human and chimpanzee were compared, it was clear that the homologous human and chimpanzee pseudogenes were much more related to each other than to their paralogous genes. However, by bringing sequences from $G B A$ to $p s G B A$, gene conversion events would have partly homogenized the $G B A-p s G B A$ tract. We estimated that at least $13 \%$ of the human $p s G B A$ sequence was, in fact, $G B A$ sequence transported by gene conversion. These tracts were fixed in all chromosomes in the sample, indicating that the gene conversion events that generated them preceded the MRCA of human variation. The homogenizing effect of gene conversion should be taken into account when estimating duplication times from the differences between $p s G B A$ and $G B A$. The calculated estimate for the duplication time at 23.4 My ago would be solely due to, at most, the $87 \%$ of the psGBA sequence not affected by gene conversion. Thus, the time estimate can be corrected for the length of the homogenized region, and a 26.9-Mya date is obtained. Besides the likely underestimate of the extent of gene conversion, this figure may have an additional downward bias. Since the duplication event and before one of the GBA copies was inactivated, both copies may have evolved under the same constraints and at the same slow rate, which would have later increased for the copy that became $p s G B A$. Because we have assumed that the substitution rate for $p s G B A$ is constant after the duplication event, we may have un- 


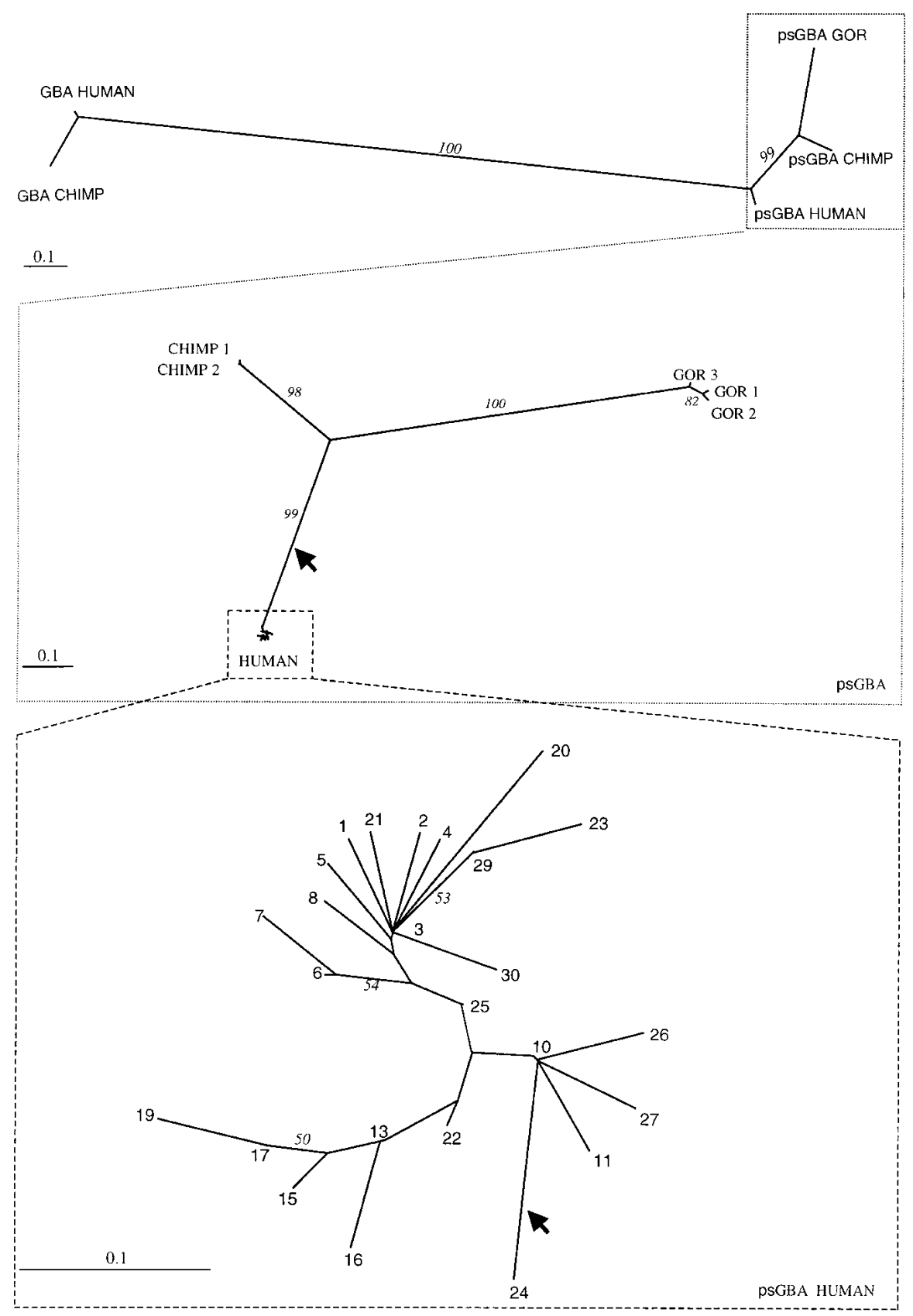

Figure 7 Neighbor-joining trees representing GBA gene and $p s G B A$ in humans, chimpanzees, and gorillas. Tree robustness was assessed by 1000 bootstrap iterations, and the percentage of trees with a given node present in the bootstrap tree is shown in the figures (italic numbers on the branches). For the lower tree, only bootstrap fractions equal or larger than $50 \%$ are shown. Arrows indicate outgroup rooting with human GBA (in the middle tree, which links human and chimpanzee psGBA sequences) and with chimpanzee psGBA (in the lower tree).

derestimated the duplication date. Our estimate is at the low end for the age range from 25 to 40 My suggested previously (Winfield et al. 1997).

\section{psGBA and Human Evolution}

The haplotype that is closest to the human MRCA sequence is, according to the haplotype phylogeny, hap- lotype 24 , which has been found only in the Biaka. The two most frequent haplotypes, which, as all other haplotypes, are more derived than 24 , are found in Africans as well as in non-Africans. This pattern, in which the most ancestral sequences are found only in Africa, has been observed repeatedly in sequences in mitochondrial DNA (Vigilant et al. 1991), autosomes (Harding et al. 1997; Clark et al. 1998), the X chromosome (Hey 1997; Zietkiewicz et al. 1997), and the Y chromosome (Shen et al. 2000), as well as in Y-chromosome polymorphisms (Hammer et al. 1995; Underhill et al. 1997). This set of observations, among others, shows coalescence times younger than $1 \mathrm{My}$, and that genetic diversity in nonAfricans is a phylogenetic subset of that in Africans, and, therefore, it is compatible with a common, recent origin of anatomically modern humans in Africa.

The structure of the haplotype phylogeny contains two haplotype groups that radiate, respectively, from haplotypes 3 and 17 . The former haplotype group has a clearer starlike structure and is in a looser linkage disequilibrium with polymorphisms at the $G B A$ gene. Both features indicate an older age for haplogroup 3 than for 17 , which is confirmed by the ages estimated for the most derived mutations that define haplotypes 3 and 17 , which are, respectively, $74,000 \pm 27,300 \mathrm{yr}$ ago for 4938 and $37,500 \pm 16,000 \mathrm{yr}$ ago for 4020 .

The TMRCA estimated for $p s G B A, \sim 199,000 \mathrm{yr}$ ago under the constant population model and $\sim 91,000 \mathrm{yr}$ ago under the growth model, is the most recent found to date for autosomal loci. Previous estimates from autosomal loci locate the TMRCA around 1 Mya (Harding et al. 1997; Clark et al. 1998). Our estimate would be closer to the age estimated recently for the Y chromo-

\section{Genome Research}


some $(50,000$ yr ago; Thomson et al. 2000), correcting for the fourfold lower population size of the Y chromosome, and for the Apolipoprotein E gene $(300,000$ yr ago; Fullerton et al. 2000). Nevertheless, one should be cautious when making inferences from genomic data to population history, because it might be that the ages we obtain are influenced more by genomic than by population events. Different genomic regions may have different evolutive histories. For instance, selection could have had an influence on shaping the psGBA variability pattern. This would shorten the psGBA gene genealogy observed currently. What is clear from the data on $p s G B A$ is that it is possible to obtain such recent ages for autosomal loci. Different coalescence ages are being obtained from different human loci. Thus, perhaps the distribution of coalescence times over a number of loci is more informative than any single-locus estimate.

In summary, we have shown the interplay of a number of forces, such as recombination, recurrent mutation, and gene conversion, in shaping the phylogeny and polymorphism of a human autosomal pseudogene. Both aspects of the dynamics of the genome region, genomic and population-based factors, have been uncovered in a complex but meaningful analysis.

\section{METHODS}

To have a global representation of the variability pattern on psGBA, five individuals from 10 populations representing all major world geographic areas were analyzed: Biaka Pygmies (from the Central African Republic) and Tanzanians (from the region of Morogoro in the South East of Tanzania), both from sub-Saharan Africa; Saharawi from Western Sahara, in North Africa; Druze from Northern Israel, in the Middle East; Basques and Catalans, both from the Iberian Peninsula, in Europe; Yakut (from Siberia) and Han Chinese, both from East Asia; Mayan from Yucatan, America; Nasioi from Bougainville Island in Melanesia, Pacific. Informed consent was obtained from all individuals included in this study. DNA from Basque, Catalan, Tanzanian, and Saharawi samples was extracted from fresh blood using a standard phenol-chloroform extraction method after digestion with proteinase K. DNA from Biaka, Mayan, Yakut, Chinese, Druze, and Nasioi was obtained from lymphoblastoid cell lines maintained in Kenneth and Judy Kidd's laboratory at Yale University. Two unrelated chimpanzees (Pan troglodytes) and two unrelated gorillas (Gorilla gorilla) were included in the sample to perform phylogenetic comparisons. In total, the precise sequence of 108 psGBA alleles has been determined, and almost $600 \mathrm{~kb}$ sequenced.

A 5.7-kb DNA segment encompassing the psGBA region was amplified using $p S G B A$-specific primers (forward primer sequence: 5 '-acatcacggtagcctcagcatgttgtg-3'; reverse primer sequence: 5 '-cccaagactggtttttctactctcatgac-3'). PCR conditions were as follows: $0.24 \mathrm{mM}$ dNTPs, $6 \times 10^{-5} \mathrm{mM}$ each primer, 200-400 ng genomic DNA, $1.5 \mathrm{mM} \mathrm{MgCl}_{2}$ buffer, 3.5 units of High Fidelity enzyme mix in $50 \mu \mathrm{L}$ final volume. The PCR profile starts with a denaturation step of 2 min at $94^{\circ} \mathrm{C}$, followed by 10 cycles of $15 \mathrm{sec}$ at $94^{\circ} \mathrm{C}, 30 \mathrm{sec}$ at $60^{\circ} \mathrm{C}, 4 \mathrm{~min}$ at $68^{\circ} \mathrm{C}, 20$ cycles with the same conditions but with $20 \mathrm{sec}$ additional elongation per cycle, and a final elongation step for $8 \mathrm{~min}$ at $72^{\circ} \mathrm{C}$. The PCR amplicon was sequenced directly on an automated sequencer (ABI PRISM 377, PE Biosystems), using the ABI PRISM dRhodamine Terminator Cycle Sequencing Ready Reaction kit with Ampli Taq Polymerase (PE Biosystems). DNA sequencing was performed using a battery of primers that yield sequencing fragments that overlap between successive reactions (details of the primers used are available on request). The chromatograms were imported into the Seqman II software (Lasergene package, DNASTAR Inc.), assembled, and analyzed. A visual screening was also performed to detect any suspected heterozygous site. Heterozygous sites were detected and the genotypes for all the individuals were obtained. A 5420-bp tract could be ascertained unambiguously for all the individuals of our sample set. PCR-amplified and sequenced regions are indicated in Figure 1. Position 1 was defined as the first nucleotide in this stretch, which corresponds to nucleotide 280 in the pseudogene sequence in Horowitz et al. (1989) (GenBank J03060). The same primer pair and PCR conditions were used to amplify $p s G B A$ from human, chimpanzee, and gorilla samples. The whole amplified segment was cloned for those samples for which haplotype assignment was not direct, that is, those with more than one heterozygous site. To discern the phase among them, tracts with heterozygous sites were resequenced in one clone from each cloned sample. The sequence of the other allele was inferred, and the phase was reconfirmed by sequencing a different clone.

The $G B A$ sequence was obtained for one chimpanzee. PCR $G B A$-specific primers were used (forward: 981-1006, reverse: 8203-8224, according to GenBank J03059 for the human $G B A$ sequence). PCR conditions were the same as those used for $p s G B A$, except that the annealing temperature was decreased from $60^{\circ} \mathrm{C}$ to $58^{\circ} \mathrm{C}$, and elongation time was increased from 4 to $8 \mathrm{~min}$. Additional inner primers, located at positions 2436-2453, 4018-4001, and 3038-3057 (numeration from $5^{\prime}$ to $3^{\prime}$ according to GenBank J03059), were used for sequencing.

Diversity parameters were calculated with the DnaSP software (DNA Sequence Polymorphism version 3.14; Rozas and Rozas 1999). Only single-nucleotide substitutions were considered in the calculations of the diversity parameters. $F_{s t}$ and haplotype ascertainment between $p s G B A$ and $G B A$ polymorphisms were estimated with the Arlequin version 2.000 package (Schneider et al. 2000). Network 2.0b analysis software was used to establish median-joining networks among the haplotypes of our sample set (Bandelt et al. 1995). Neighbor-joining trees (Saitou and Nei 1987) were built from a sequence distance matrix computed with the DNADIST program, in the Phylip 3.5c package (Felsenstein 1989). The SITES program (http://heylab.rutgers.edu/) was used to compute the $\gamma$ recombination parameter. The GENETREE program was used to estimate coalescence times and the age of mutations (Griffiths and Tavaré 1994; 1998a; 1998b). In addition, we calculated an independent estimate of the age of haplogroup 3; considering a constant-rate neutral mutation process, the number of mutations that would have accumulated in a given sample of sequences springing from a common ancestral sequence follows a Poisson distribution with mean $\lambda=\mu \mathrm{t}$, where $\mu$ is the mutation rate per segment and per year and $t$ is the time elapsed since the coalescence of all the sequences. We can estimate $\lambda$ as an average number of differences from the ancestral sequence in haplogroup 3 (Bertranpetit and Calafell 1996). The standard error of $\lambda$ was estimated 
as $(\lambda / n)^{1 / 2}$, where $n$ is the number of chromosomes considered (Rando et al. 1998).

\section{ACKNOWLEDGMENTS}

We are indebted to K.M. Weiss and A. Buchanan for technical assistance and helpful comments on an earlier version of the manuscript. We thank Kenneth K. Kidd, Judith R. Kidd, and B. Bonné-Tamir for sharing DNA samples. This research was supported by Dirección General de Investigación Científica y Técnica (Spanish Government) grant PB98-1064, and by Generalitat de Catalunya, Grup de Recerca Consolidat 1998SGR00009. R.M.-A. received a fellowship from the Spanish Ministry of Education and Culture (AP96).

The publication costs of this article were defrayed in part by payment of page charges. This article must therefore be hereby marked "advertisement" in accordance with 18 USC section 1734 solely to indicate this fact.

\section{REFERENCES}

Bandelt, H., Forster, P., Sykes, B.C., and Richards, M.B. 1995. Mitochondrial portraits of human populations using median networks. Genetics 141: 743-753.

Barbujani, G., Magagni, A., Minch, E., and Cavalli-Sforza, L.1997. An appointment of human DNA diversity. Proc. Natl. Acad. Sci. 94: 4516-4519.

Bertranpetit, J. and Calafell, F. 1996. Genetic and geographical variability in cystic fibrosis: Evolutionary considerations. In Variation in the human genome, pp 97-118. (ed. G. Cardew) Wiley, Chichester (Ciba Foundation Symposium 197).

Beutler, E., West, C., and Gelbart, T. 1992. Polymorphisms in the human glucocerebrosidase gene. Genomics 12: 795-800.

Broman, K.W. and Weber, J.L. 2000. Characterization of human crossover interference. Am. J. Hum. Genet. 66: 1911-1926.

Clark, G.A., Weiss, K.M., Nickerson, D.A., Taylor, S.L., Buchanan, A., Stengard, J., Salomaa, V., Vartiainen, E., Perola, M., Boerwinkle, E., et al. 1998. Haplotype structure and population genetic inferences from nucleotide-sequence variation in human lipoprotein lipase. Am. J. Hum. Genet. 63: 595-612.

Cooper, D.N. 1999. Pseudogenes and their formation. In Human Gene Evolution (ed. D.N. Cooper), pp 265-296. BIOS Scientifics Publishers Ltd., Oxford.

Devine, E.A., Smith, M., Arredongo-Vega, F.X., Shafit-Zagardo, B., and Desnick, R.J. 1982. Regional assignment of the structural gene for human acid $\beta$-glucosidase to $\mathrm{q} 42 \rightarrow \mathrm{qter}$ on chromosome 1. Cytogenet. Cell Genet. 33: 340-344.

Dorit, R.L., Akashi, H., and Gilbert, W. 1995. Absence of polymorphisms at the ZFY locus on the human Y chromosome. Science 268: 1183-1185.

Felsenstein, J. 1989. PHYLIP-phylogeny inference package (version 3.2). Cladistics 5: 164-166.

Fu, Y.X. and Li, W.H. 1993. Statistical tests of neutrality of mutations. Genetics 133: 693-709.

Fullerton, S.M., Harding, R.M., Boyce, A.J., and Clegg, J.B. 1994. Molecular and population genetic analysis of allelic sequence diversity at the human $\beta$-globin locus. Proc. Natl. Acad. Sci. 91: $1805-1809$.

Fullerton, S.M., Clark, A.G., Weiss, K.M., Nickerson, D.A., Taylor, S.T., Stengård, J.H., Salomaa, V., Vartiainen, E., Perola, M., Boerwinkle, E., et al. 2000. Apolipoprotein E variation at the sequence haplotype level: Implications for the origin and maintenance of a major human polymorphism. Am. J. Hum. Genet. 67: 881-900.

Ginns, E., Choudary, P.V., Tsuji, S., Martin, B., Stubblefield, B., Sawyer, J., Hozier, J., and Barranger, J. 1985. Gene mapping and leader polypeptide sequence of human glucocerebrosidase: Implications for Gaucher disease. Proc. Natl. Acad. Sci. 82: 7101-7105.
Glenn, D., Gelbart, T., and Beutler, E. 1994. Tight linkage of pyruvate kinase (PKLR) and glucocerebrosidase (GBA) genes. Hum. Genet. 93: 635-638.

Griffiths, R.C. and Tavaré, S. 1994. Ancestral inference in population genetics. Stat. Sci. 9: 307-319.

-1998a. The age of a mutation in a general coalescent tree. Commun. Stat. 14: 273-295.

- 1998b. The ages of mutations in gene trees. Ann. Appl. Prob. 9: $567-590$.

Grimsley, C., Mather, K.A., and Ober, C. 1998. HLA-H: A pseudogene with increased variation due to balancing selection at neighboring loci. Mol. Biol. Evol. 15: 1581-1588.

Halushka, M.K., Fan, J.-B., Bentley, K., Hsie, L., Shen, N., Weder, A., Cooper, R., Lipshutz, R., and Chakravarti, A. 1999. Patterns of single-nucleotide polymorphisms in candidate genes for blood-pressure homeostasis. Nat. Genet. 22: 239-247.

Hammer, M.F. 1995. A recent common ancestry for human Y chromosomes. Nature 378: 376-378.

Harding, R.M., Fullerton, S.M., Griffiths, R.C., Bond, J., Cox, M.J., Schneider, J.A., Moulin, D.S., and Clegg J.B. 1997. Archaic African and Asian lineages in the genetic ancestry of modern humans. Am. J. Hum. Genet. 60: 772-789.

Harris, E.E. and Hey, J. 1999. X chromosome evidence for ancient human histories. Proc. Natl. Acad. Sci. 96: 3320-3324.

Hey, J. 1997. Mitochondrial and nuclear genes present conflicting portraits of human origins. Mol. Biol. Evol. 14: 166-172.

Hey, J. and Wakeley, J. 1997. A coalescent estimator of the population recombination rate. Genetics 145: 833-846.

Hong, C.M., Ohashi, T., Yu, X.Y., Weiler, S., and Barranger, J.A. 1990. Sequence of two alleles responsible for Gaucher disease. DNA Cell Biol. 9: 233-241.

Horowitz, M., Wilder, S., Horowitz, Z., Reiner, O., Gelbart, T., and Beutler, E. 1989. The human glucocerebrosidase gene and pseudogene: Structure and evolution. Genomics 4: 87-96.

Hudson, R.R. 1987. Estimating the recombination parameter of a finite population model without selection. Genet. Res. 50: $245-250$

Hudson, R. and Kaplan, N.L. 1985. Statistical properties of the number of recombination events in the history of a sample of DNA sequences. Genetics 111: 147-164.

Imai, K., Nakamura, M., Yamada, M., Asano, A., Yokoyama, S., Tsuji, S., and Ginns, E. 1993. A novel transcript from a pseudogene for human glucocerebrosidase in non-Gaucher disease cells. Gene 136: $365-368$.

Jaruzelska, J., Zietkiewicz, E., Batzer, M., Cole, D.E.C., Moisan, J.P., Scozzari, R., Tavaré, S., and Labuda, D. 1999a. Spatial and temporal distribution of the neutral polymorphisms in the last ZFX intron: Analysis of the haplotype structure and genealogy. Genetics 152: 1091-1101.

Jaruzelska, J., Zietkiewicz, E., and Labuda, D. 1999b. Is selection responsible for the low level of variation in the last intron of the ZFY locus? Mol. Biol. Evol. 16: 1633-1640.

Jorde, L.B., Watkins, W.S., Bamshad, M.J., Dixon, M.E., Ricker, C.E., Seielstad, M.T., and Batzer, M.A. 2000. The distribution of human genetic diversity: A comparison of mitochondrial, autosomal, and Y-chromosome data. Am. J. Hum. Genet. 66: 979-988.

Kaessman, H., Heißig, F., Haeseler, A., and Pääbo, S. 1999. DNA sequence variation in a non-coding region of low recombination on the human X chromosome. Nat. Genet. 22: 78-81.

Koprivica, V., Stone, D.L., Park, J.K., Callahan, M., Frisch, A., Cohen, I.J., Tayebi, N., and Sidransky, E. 2000. Analysis and classification of 304 mutant alleles in patients with type 1 and type 3 Gaucher disease. Am. J. Hum. Genet. 66: 1777-1786.

Latham, T.E., Theophilus, B.D.M., Grabowski, G.A., and Smith, F.I. 1991. Heterogeneity of mutations in the acid $\beta$-glucosidase gene of Gaucher disease patients. DNA Cell Biol. 10: 15-21.

Li, W.-H. and Sadler, L.A. 1991. Low nucleotide diversity in man. Genetics 129: 513-523.

Li, W.-H., Gojobori, T., and Nei, M. 1981. Pseudogenes as a paradigm of neutral evolution. Nature 292: 237-239. 
Nachman, M.W., Bauer, V.L., Crowell, S.L., and Charles, F.A. 1998. DNA variability and recombination rates at X-linked loci in humans. Genetics 150: 1133-1141.

Nei, M. and Li, W.-H. 1979. Mathematical model for studying genetic variation in terms of restriction endonucleases. Genetics 76: 5369-5273.

Rana, B.K., Hewett-Emmett, D., Jin, L., Chang, B.H., Sambuughin, N., Lin, M., Watkins, S., Bamshad, M., Jorde, L.B., Ramsay, M., et al. 1999. High polymorphism at the human melanocortin 1 receptor locus. Genetics 151: 1547-1557.

Rando, J.C., Pinto, F., González, A.M., Hernández, M., Larruga, J.M., Cabrera, V.M., and Bandelt, H.-J. 1998. Mitochondrial DNA analysis of Northwest African populations reveals genetic exchange with European, Near-Eastern, and sub-Saharan populations. Ann. Hum. Genet. 62: 531-550.

Reiner, O. and Horowitz, M. 1988. Differential expression of the human glucocerebrosidase-coding gene. Gene 17: 469-478.

Rieder, M.J., Taylor, S.L., Clark, A.G., and Nickerson, N.A. 1999. Sequence variation in the human angiotensin converting enzyme. Nat. Genet. 22: 59-62.

Rozas, J. and Rozas, R. 1999. DnaSP version 3: An integrated program for molecular population genetics and molecular evolution analysis. Bioinformatics 15: 174-175.

Saitou, N. and Nei, M. 1987. The neighbour-joining method: A new method for reconstructing phylogenetic trees. Mol. Biol. Evol. 4: $406-425$.

Schneider, S., Kueffer, J.M., Roessli, D., and Excoffier, L. 2000. Arlequin ver.2000: A software environment for the analysis of population genetics data. Geneva, Genetics and Biometry Laboratory, University of Geneva, Switzerland.

Shafit-Zagardo, B., Devine, E.A., Smith, M., Arredongo-Vega, F., and Desnick, R.J. 1981. Assignment of the gene for acid $\beta$-glucosidase to human chromosome 1. Am. J. Hum. Genet 33: 564-575.

Shen, P., Wang, F., Underhill, P.A., Franco, C., Yang, W.-H., Roxas, A., Sung, R., Lin, A.A., Hyman, R.W., Vollrath, D., et al. 2000. Population genetic implications from sequence variation in four Y chromosome genes. Proc. Natl. Acad. Sci. 97: 7354-7359.

Simonsen, K.L., Churchill, G.A., and Aquadro, C.F. 1995. Properties of statistical tests of neutrality for DNA polymorphism data. Genetics 141: 413-429.

Sorge, J., West, C., Westwood, B., and Beutler, E. 1985. Molecular cloning and nucleotide sequence of human glucocerebrosidase cDNA. Proc. Natl. Acad. Sci. 82: 7289-7293.
Sorge, J., Gross, E., West, C., and Beutler, B. 1990. High level transcription of the glucocerebrosidase pseudogene in normal subjects and patients with Gaucher disease. J. Clin. Invest. 86: $1137-1141$

Stone, D.L., Tayebi, N., Orvisky, E., Stubblefield, B., Madike, V., and Sidransky, E. 2000. Glucocerebrosidase gene mutations in patients with type 2 Gaucher disease. Hum. Mutat. 15: 181-188.

Tajima, F. 1989. Statistical method for testing the neutral mutation hypothesis. Genetics 123: 585-595.

Thomson, R., Pritchard, J.K., Shen, P., Oefner, P.J., and Feldman, M.W. 2000. Recent common ancestry of human Y chromosome: Evidence from DNA sequence data. Proc. Natl. Acad. Sci.97: 7360-7365.

Underhill, P.A., Jin, L., Lin, A.A., Mehdi, S.Q., Jenkins, T., Vollrath, D., Davis, R.W., Cavalli-Sforza, L.L., and Oefner, P.J. 1997. Detection of numerous Y chromosome biallelic polymorphisms by denaturing high-performance liquid chromatography. Genome Res. 7: 996-1005.

Vigilant, L., Stoneking, M., Harpending, H., Hawkes, K, and Wilson, A.C. 1991. African populations and the evolution of human mitochondrial DNA. Science 253: 1503-1507.

Watterson, G.A. 1975. On the number of segregating sites in genetical models without recombination. Theor. Popul. Biol. 7: 256-276.

Whitfield, L.S., Sulston, J.E., and Goodfellow, P.N. 1995. Sequence variation of the human Y chromosome. Nature 378: 379-380.

Winfield, S.L., Tayebi, N., Martin, B.M., Ginns, E.I., and Sidransky, E. 1997. Identification of three additional genes contiguous to the glucocerebrosidase locus on chromosome 1q21: Implications for Gaucher disease. Genome Res. 7: 1020-1026.

Zietkiewicz, E., Yotova, V., Jarnik, M., Korab-Laskowska, M., Kidd, K.K., Modiano, D., Scozzari, R., Stoneking, M., Tishkoff, S., Batzer, M., et al. 1997. Nuclear DNA diversity in worldwide distributed human populations. Gene 205: 161-171.

- 1998. Genetic structure of the ancestral population of modern humans. J. Mol. Evol. 47: 146-155.

Zimran, A., Sorge, J., Gross, E., Kubitz, M., West, C., and Beutler, E. 1990. A glucocerebrosidase fusion gene in Gaucher disease. J. Clin. Invest. 85: 219-222.

Received October 16, 2000; accepted in revised form February 28, 2001.
Genome Research www.genome.org 


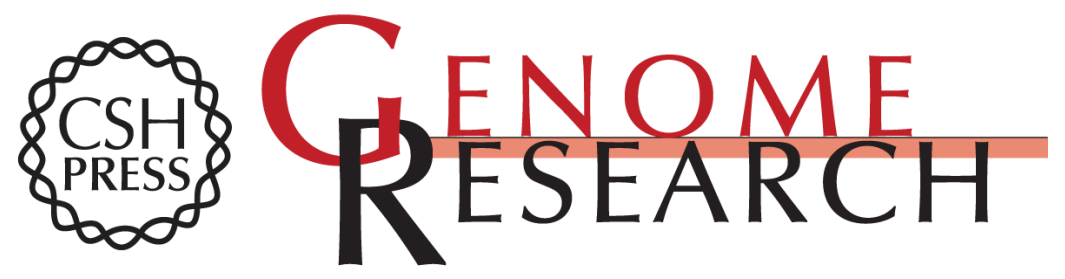

\section{Sequence Variability of a Human Pseudogene}

Rosa Martínez-Arias, Francesc Calafell, Eva Mateu, et al.

Genome Res. 2001 11: 1071-1085

Access the most recent version at doi:10.1101/gr.167701

\section{License}

Email Alerting Receive free email alerts when new articles cite this article - sign up in the box at the Service top right corner of the article or click here.

\section{Affordable, Accurate Sequencing.}

To subscribe to Genome Research go to: https://genome.cshlp.org/subscriptions 